\title{
A imagética motora no desempenho funcional de pacientes com esclerose múltipla: revisão de literatura
}

\section{Motor imagery in the functional performance of patients with multiple sclerosis: literature review}

\author{
Tassiane Maria Alves Pereira ${ }^{\dagger}$, Letícia de Sousa Vidal, Marco Orsini ${ }^{\S}$, Janaína Silvall
}

Como citar esse artigo. Pereira, T. M.

A.; Vidal, L. S.; Orsini, M.; Silva, J.

A imagética motora no desempenho funcional de pacientes com esclerose múltipla: revisão de literatura. Revista de Saúde. 2020 Jul./Dez.; 11 (2): 55 - 59.

\begin{abstract}
Resumo
A Esclerose Múltipla é uma doença neurodegenerativa incapacitante oriundas de danos ao Sistema Nervoso Central. Entre os sinais e sintomas destacam-se os comprometimentos motores, sensoriais e emocionais que interferem negativamente na qualidade de vida desses pacientes. A Imagética Motora é uma terapia usada através da simulação mental do ato motor capaz de promover alterações neurofisiológicas e melhorar o desempenho funcional. O objetivo desta revisão é verificar o efeito da imagética motora no desempenho funcional de pacientes com esclerose múltipla. Dessa forma, realizou-se uma busca nas bases e banco de dados Pubmed, PEDro, Scopus e Web of Science utilizando os descritores "motor imagery" AND "multiple sclerosis" AND "functionality". estipulando critérios de inclusão e de qualidades metodológicas. No total 5 artigos foram incluídos e compuseram a análise da revisão. Os resultados evidenciaram melhoras significativas na marcha, equilíbrio, qualidade da caminhada, fadiga e qualidade de vida desses pacientes. Conclui-se então que a imagética motora é uma intervenção eficaz na reabilitação funcional de pacientes acometidos pela Esclerose Múltipla.

Palavras-chave: Imagética Motora, Esclerose Múltipla, Funcionalidade, Fisioterapia.
\end{abstract} rehabilitation of patients affected by Multiple Sclerosis.

Keywords: Motor Imagery, Multiple Sclerosis, Functionality, Physiotherapy.

\section{Introdução}

A Esclerose Múltipla (EM) é uma doença neurológica, inflamatória, progressiva e incapacitante decorrente do ataque autoimune à substância branca do Sistema Nervoso Central ${ }^{1,2,3}$. Caracteriza-se por episódios de exacerbação da atividade inflamatória, chamados de surtos que alternam em períodos de remissão, progressão e a combinação dos dois aspectos, que classificam os cursos clínicos em: recorrente- remitente, progressiva secundária, progressiva primária e progressiva-recorrente. A EM é a causa importante de incapacidade física grave em adultos jovens, apresentando sintomas geralmente no final dos 20 anos, sendo mais incidente no sexo feminino ${ }^{3-8}$.

Os sinais e sintomas da EM mais frequente estão relacionados a fadiga, espasticidade, comprometimentos motores, sensoriais, visuais, esfincterianos, emocionais e sexuais. Estudos reportam que 40 a $80 \%$ dos pacientes relatam fadiga associada a dificuldade de caminhar,

Afiliação dos autores: ${ }^{\dagger}$ Centro Universitário UNINASSAU, Teresina - PI, Brasil; Pós-graduanda em Fisioterapia Hospitalar pela Inspirar, Teresina-PI, Brasil; Mestranda em Biotecnologia - UFPI/UFDPAR, Parnaíba-PI, Brasil. https://orcid.org/0000-0002-8876-7438

†Universidade Estadual do Piauí (UESPI), Teresina - PI, Brasil. https://orcid.org/0000-0001-6988-8798

${ }^{\S}$ Universidade Iguaçu- UNIG-RJ, Nova Iguaçu - RJ, Brasil; Universidades de Vassouras, Vassouras, Rio de Janeiro - RJ, Brasil. https://orcid.org/0000-0002-8526-6937

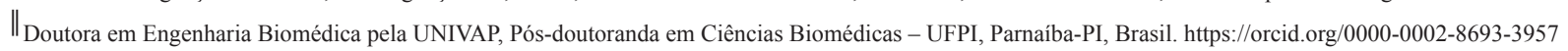

* Email de correspondência: fisiojanainams@gmail.com

Recebido em: 22/05/20. Aceito em: 05/09/20. 
o que influencia no seu desempenho funcional e independência para a realização de atividades de vida diária9 $^{-11}$.

O comprometimento funcional dos pacientes com EM levam a uma série de condições cognitivas e emocionais que afetam consideravelmente sua qualidade de vida. Nesta perspectiva, existem diversas intervenções que visam promover melhoras no desempenho funcional e bem-estar global desses pacientes. Entre elas está a Imagética Motora (IM) que tem sido um recurso promissor e de fácil adesão para o tratamento de déficits neurológicos ${ }^{11-16}$.

A IM trata-se da simulação mental de um ato motor, na ausência de qualquer ativação muscular, podendo ser entendido como contendo duas estratégias principais: visual, onde o indivíduo imagina outra pessoa realizando o movimento, e cinestésica em que o indivíduo se sente executando o movimento. Esta técnica de treinamento é capaz de gerar adaptações neurofisiológicas por ser capaz de ativar as mesmas regiões do encéfalo que a execução física da tarefa, e além de gerar reorganização cortical, também influencia na ativação dos músculos periféricos por ser capaz de modular a excitabilidade corticoespinhal ${ }^{16-19}$.

Tendo em vista os déficits funcionais decorrentes da EM, torna-se necessário a investigação da eficácia de novas formas de tratamento que objetivem a reabilitação funcional desses pacientes. Dessa forma, o objetivo deste estudo foi revisar na literatura evidências que apontem o efeito da imagética motora no desempenho funcional de pacientes com Esclerose Múltipla.

\section{Material e Métodos}

O estudo tratou-se de uma revisão de literatura através da busca nos bancos e bases de dados PubMed, PEDro, Scopus e Web of Science baseado na estratégia PICO (P-population: adultos diagnosticados com Esclerose Múltipla; I-Intervencion: Imagética Motora; C-Comparation: não se aplica a este estudo; O: Outcomes: Melhora do desempenho funcional) para formulação da pergunta norteada da pesquisa "Como a Imagética motora influencia na funcionalidade dos pacientes com Esclerose Múltipla?" e elaboração dos descritores "motor imagery" AND "multiple sclerosis" AND "functionality".

Foram selecionados artigos com ensaios clínicos controlados randomizados, publicados nos últimos 5 anos nos idiomas português e inglês, que utilizaram a Imagética motora na reabilitação de pacientes com diagnóstico de Esclerose Múltipla. Foram excluídos artigos duplicados, que fugiam ao tema e que apresentavam desenhos de pesquisa inapropriados.

A busca e seleção foram realizadas por dois revisores independentes inicialmente através da leitura do título e posteriormente a leitura dos resumos para identificação do tipo de estudo e critérios de inclusão utilizados. As discordâncias entre os revisores durante a análise foram decididas por consenso.

Para a avaliação da qualidade metodológica dos artigos selecionados foi utilizada a escala PEDro que qualifica os ensaios clínicos controlados randomizados seguindo 11 critérios com pontuação de 1 a 10 , sendo o primeiro critério não pontuado. Estudos com pontuação maior ou igual a 6 são considerados de alta qualidade.

\section{Resultados}

Foram encontrados 108 artigos por meio das bases de dados pesquisadas, 101 foram excluídos por fugirem ao tema, apresentarem desenhos e ano de publicação inapropriados ou serem duplicados. Assim 7 estudos foram incluídos para uma avaliação criteriosa, destes 5 contemplavam os critérios de inclusão adequados (Figura 1).

Os artigos incluídos observaram o efeito do IM no desempenho funcional de pacientes com EM. Foram analisados 267 pacientes de 18 a 65 anos com diagnóstico clínico de Esclerose Múltipla avaliando marcha e equilíbrio na caminhada (Índice de Marcha Dinâmica), velocidade e resistência na marcha (Caminhada cronometrada de 25 pés- $\mathrm{T} 25 \mathrm{FW}$ ) e habilidade percebida (MS Walking Scale - MSWS-12), habilidade de IM (Kinesthetic and Visual Imagery Questionnarie-

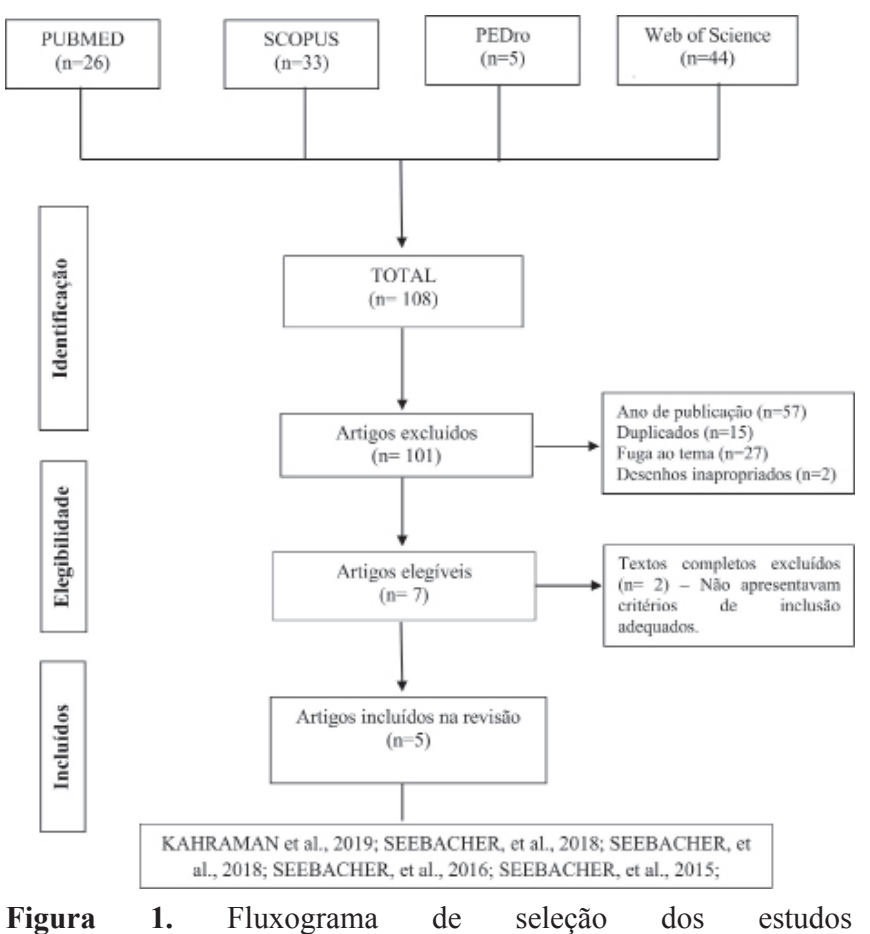


KVIQ) ${ }^{11,12}$, desempenho do equilíbrio (TUGtest, Scale Balance Confidence) ${ }^{12}$, fadiga (Escala de Impacto de fadiga Modificada - MFIS) $)^{11}$ - 15 , velocidade da marcha (Timed 25- Foot Walk)e distância a pé (6 - Minute Walk Test $)^{11,13,14,15}$, percepção da caminhada ${ }^{14}$, ansiedade e depressão ${ }^{12}$, qualidade de vida ${ }^{12-14}$, taxa de recrutamento, retenção, adesão e eventos adversos ${ }^{11,15}$ (Quadro 1).

\section{Discussão}

Este estudo veio expor evidências sobre o efeito da imagética motora no desempenho funcional de pacientes

Quadro 1. Resumo dos estudos incluídos apresentando o tamanho da amostra, a forma de aplicação da intervenção, os desfechos e resultados obtidos na população estudada.

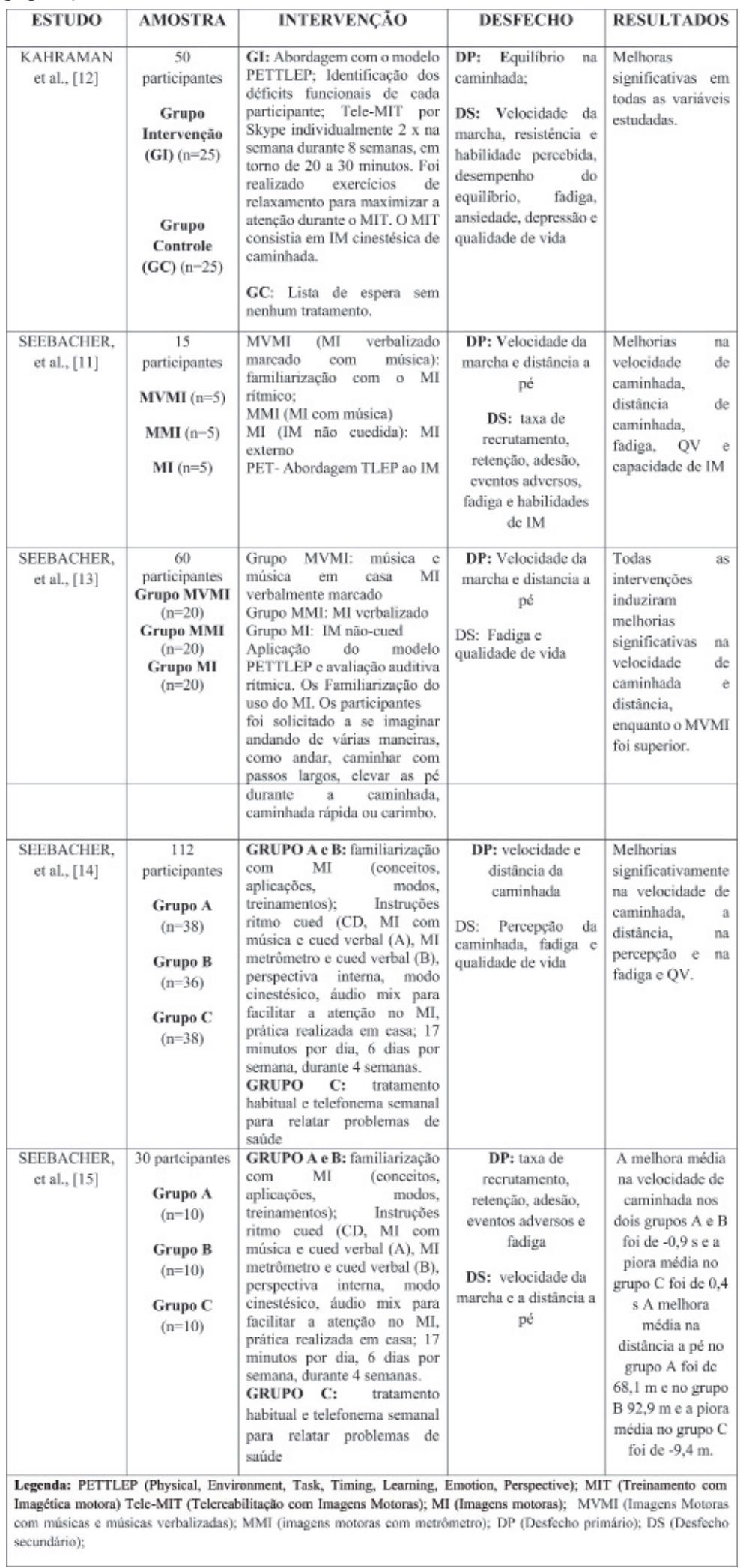


com Esclerose Múltipla. Estudos evidenciaram melhoras significativas no desempenho da caminhada o que inclui a qualidade, resistência e velocidade da marcha, equilíbrio na caminhada e percepção da caminhada ${ }^{11-}$ 15. A recuperação motora em indivíduos submetidos a imagética pode estar relacionada a particularidade da aprendizagem na reabilitação motora e a semelhança neural com o movimento por apresentar os mesmos processos corticais envolvidos nas contrações reais, principalmente na realização da imagética cinestésica, envolvendo a ativação dos córtices pré-motores e motores primários, área motora suplementar e vias córtico-espinal ${ }^{19-22}$.

Com relação a habilidade de IM, a proficiência na sua realização observadas através do Questionário de Imagens motoras visuais e cinestésicas garantem que estes pacientes apresentaram um bom desempenho na prática de IM assegurando a eficácia do treinamento ${ }^{11,12}$, embora a EM favoreça alterações cognitivas que possam interferir na qualidade, interpretação e execução da $\mathrm{IM}^{19}$. Contudo, a eficiência na realização de IM favorece a efetivação do planejamento motor o que facilita o aprendizado e ganhos no desempenho físico ${ }^{12,23,24}$.

A fadiga se destaca como um dos principais sintomas relacionados a perda do desempenho funcional em portadores de EM. Estudos revelaram melhoras na fadiga física e cognitiva após treino com $\mathrm{IM}^{11-15}$, o que pode estar relacionado a boa adesão ao treinamento e a realização do movimento sem esforço físico, além da repercussão cortical mediante a IM que pode favorecer o aumento de ganhos funcionais e redução desse sintoma $^{19,25}$

A EM afeta negativamente a qualidade de vida destes pacientes por ocasionar redução das habilidades físicas ${ }^{5,19,24}$. Ao avaliar a qualidade de vida após o treino de IM, estudos evidenciaram aumentos significativos nos scores de avaliação $^{12,13,14}$ enfatizando o avanço no desempenho funcional e bem estar global desses indivíduos potencializando a capacidade de ser independente e levar uma vida saudável ${ }^{15,19}$.

As limitações deste estudo estão relacionadas a quantidade de ensaios clínicos utilizando imagética motora neste público e a não caracterização do curso clínico da doença utilizando assim o mesmo protocolo para todos os casos, o que pode não evidenciar os resultados reais da IM em todos os casos.

\section{Considerações Finais}

Diante das evidências expostas, foi possível observar o efeito do treinamento com imagética motora em pacientes com Esclerose Múltipla apresentando melhoras em parâmetros que potencializam o seu desempenho funcional favorecendo a funcionalidade e independência, podendo ainda, servir de estratégia de reabilitação eficaz e promissora no tratamento destes pacientes.

\section{Referências}

1. KLOCKE S, HAHN N. Multiple sclerosis. Ment Health Clin. 2019;9(6):349 $\square 358$

2. MACARON G, ONTANEDA D. Diagnosis and Management of Progressive Multiple Sclerosis. Biomedicines. 2019;7(3):56.

3. COPTSAPAS C, MITROVIC M, HAFLER D. Chapter 46 - Multiple sclerosis. Handbook of Clinical Neurology 2018;148:723-730.

4. GOSSMAN W, EHSAN M, XIXIS KL. Multiple Sclerosis. In: StatPearls. Treasure Island (FL): StatPearls Publishing; 2020

5. NEVES, Conceição Fernandes da Silva, et al. "Qualidade de vida da pessoa com esclerose múltipla e dos seus cuidadores.” Revista de Enfermagem Referência 12 (2017): 85-96.

6. GUIMARÃES, J., \& SÁ, M. J. (2014). Esclerose múltipla e outras doenças inflamatórias e desmielinizantes do sistema nervoso central. In M. J. Sá (Coord.), Neurologia clínica: Compreender as doenças neurológicas ( $2^{\mathrm{a}}$ ed., pp. 373-411). Porto, Portugal: Edições Universidade Fernando Pessoa.

7. KAMISNKA J, KOPER OM, PIECHAL K, KEMONA H; Multiple sclerosis - etiology and diagnostic potential. Med Dosw 2017; 71 : 551-563.

8. CORREALE J, GAITÁN MI, YSRRAELIT MC, FIOL MP. Progressive multiple sclerosis: from pathogenic mechanisms to treatment. Brain 2017; 140:527-546.

9. KRUPP L. Fatigue is intrinsic to multiple sclerosis (MS) and is the most commonly reported symptom of the disease. Mult Scler. 2006;12:367-8.

10. INDURUWA I, CONSTANTINESCU CS, GRAN B. Fatigue in multiple sclerosis -a brief review. J Neurol Sci. 2012; 323:9-15.

11. SEEBACHER B, KUISMA R, GLYNN A, BERGER T. Exploring cued and non-cued motor imagery interventions in people with multiple sclerosis: a randomized reasibility trial and reliability study. Arch Physiother 2018; 8: 6

12. KAHRAMAN T, SAVCI S, OZDOGAR AT, GEDIK Z, IDIMAN E. Physical, cognitive and psychosocial effects of telerehabilitation-based motor imagery training in people with multiple sclerosis: A randomized controlled pilot trial. J Telemed Telecare. 2019; 1357633X18822355.

13. SEEBACHER B, KUISMA R, GLYNN A, BERGER T, Effects and mechanisms of differently cued and non-cued motor imagery in people with multiple sclerosis: A randomised controlled trial. Mult Scler. 2019;25(12):1593 $\square 1604$

14. SEEBACHER B, KUISMA R, GLYNN A, BERGER T. The effect of rhythmic-cued motor imagery on walking, fatigue and quality of life in people with multiple sclerosis: a randomised controlled trial. Mult Scler 2017; 23: 286-296.

15. SEEBACHER B, KUISMA R, GLYNN A, BERGER T. Rhythmic cued motor imagery and walking in people with multiple sclerosis: a randomised controlled feasibility study. Pilot Feasibility Stud. 2015;1:25.

16. BRUNO V, FOSSATARO C, GARBARINI,F; Inhibition or facilitation? Modulation of corticospinal excitability during motor imagery. Neuropsychologia, 2018;111:60-368.

17. SOUSA, Pedro Henrique Côrtes de. Análise da utilização da prática mental na recuperação funcional de indivíduos com paralisia facial periférica. 2017. xiii, 94 f., il. Dissertação (Mestrado em Ciências e Tecnologias em Saúde) - Universidade de Brasília, Brasília.

18. LEBON F, HORN U, LOTZE M; Motor imagery training: Kinesthetic imagery strategy and inferior parietal fMRI activation. Hum Brain Mapp,2018; 1-9.

19. HANSON M, CONCIALDI M; Motor imagery in multiple sclerosis: exploring applications in therapeutic Treatment. J Neurophysiol 2019;121: 347-349. 
20. GROSPRÊTRE S, RUFFINO C, LEBON F; Motor imagery and corticospinal excitability: A review. European Journal of Sport Science 2016;16 (3): $317-324$.

21. SOUZA, NS, MARTINS, AC, SAMARY, CS; Use of Motor Imagery in Modulating Cardiopulmonary Activity: Future Perspectives. Acta Neurophysiologica 2019; 1(1).

22. SOUZA, NS, MARTINS, ACG, GMMV, et al.; Efeito da imagética motora sobre o controle postural. Fisioterapia Brasil 2013; 4(14):394-397.

23. NICHOLSON V, WATTS N, CHANI Y, KEOGH JW. Motor imagery training improves balance and mobility outcomes in older adults: a systematic review. J Physiother. 2019;65(4):200 $\square 207$.

24. HEREMANS E, D'HOOGE AM, DE BONDT S, HELSEN W, FEYS P.

The relation between cognitive and motor dysfunction and motor imagery ability in patients with multiple sclerosis. Mult Scler 2012; 18: 1303-1309.

25. SOBIERAJEWICZ, J., PRZEKORACKA-KRAWCZYK, A., JASKOWSKI, W. et al. The influence of motor imagery on the learning of a fine hand motor skill. Exp Brain Res. 2017; 235. 